\title{
The MacCool/West Point
}

\author{
M. R. F. SMYTH
}

Dedicated to T.T. West on his retirement.

The serious intent behind what follows was to establish an isomorphism between two apparently unrelated questions. The idea came from a story called "Tantalising Triangles" which had been written almost thirty years ago and then consigned to a bottom drawer. It considered the following two questions:

Q1 If $A B C$ is a triangle with sides $x, y, z$ find the point $M$ that minimises $L=M A+M B+M C$.

Q2 If $x, y, z$ are the distances from any point to the vertices of an e-triangle of side $L$, express $L$ in terms of $x, y$ and $z$.

Here, and throughout this paper, e-triangle shall mean "equilateral triangle".

Let $\Delta$ be the area of the triangle with sides $x, y, z$. Unless an angle of $\triangle A B C$ exceeds $120^{\circ}$ the same answer

$$
L=\sqrt{\frac{1}{2}\left(x^{2}+y^{2}+z^{2}+4 \Delta \sqrt{3}\right)}
$$

applies in both cases; otherwise in Q1 the answer $L$ is the sum of the two shorter sides.

A primary objective of Tantalising Triangles was to stress the importance of always using the full bag of tricks. It used a rich mix of algebra, geometry, number theory, and computation, each making its own contribution to an understanding of the problem. However since it was written another hugely significant resource has emerged, namely the world-wide-web (or Web for short), and this article is based on the fresh material and insight that that has provided.

Given any triangle the point $M$ yielding the answer to Q1 is clearly one of great military significance. It is important to move and provision armies as efficiently as possible, and this point is key to doing so. Yet somehow it evaded the ancient Greeks. The great triangle 
centres familiar to them were the centroid, orthocentre, incentre, and circumcentre, and all of today's mathematicians learn about these at an early age. Strangely it wasn't until almost two thousand years later in the 17 th century that the fifth centre $M$ was eventually uncovered.

The history of this famous point can be easily traced through the Web. The earliest reference to it is attributed to Fermat. He issued Q1 as a challenge to his peers, but he didn't answer it himself. The first solution is attributed to Torricelli (of barometer fame), and the problem was generalised to networks by Steiner. As a result $M$ acquired a variety of different names; in France it was the Fermat point, in Italy the Torricelli point, and in Switzerland the Steiner point. However when its fame crossed the Atlantic something rather odd happened. It is a routine, if tedious, exercise (involving an appropriate zoning of the plane and repeated applications of the triangle inequality) to show that $M$ cannot lie outside $\triangle A B C$. The Americans identified a point that they called the First Isogonic Center which always coincided with $M$ inside but never outside of $\triangle A B C$. Approximately a century later the 9-point centre became the sixth entry in the series, and in the 19th century the quest for further triangle centres became a research area and their numbers duly mushroomed. Today the Kimberling classification lists over 3,000 triangle centres and the First Isogonic Center appears simply as $X 13$.

Superficially the ancient Greeks had a good excuse for overlooking $M$ since one might think that any solution to Q1 could require the then unknown sledgehammers of calculus and trigonometry. However the following simple argument illustrates that these tools are unnecessary.

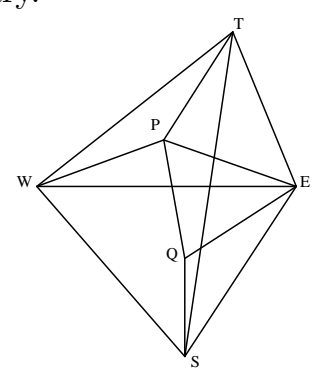

Let $W E T$ be a triangle and construct the e-triangle $W E S$ where $S$ and $T$ are on opposite sides of $W E$. Set $d(P)=W P+E P+T P$ 
for $P$ inside $\triangle W E T$ and rotate $\triangle W P E$ by $60^{\circ}$ about $E$ so that $W E$ maps onto $S E$. Let $Q$ be the image of $P$ under this rotation. Then $\triangle E P Q$ is an e-triangle so $d(P)=S Q+Q P+P T \geq S T$ with equality iff both $P$ and $Q$ lie on $S T$. Assume no angle in $\triangle W E T$ exceeds $120^{\circ}$. Then there exists $M$ on $S T$ such that $\angle T M E=120^{\circ}$. Now move $P$ to $M$ keeping $Q$ as its image under $60^{\circ}$ rotation. Although $\triangle E P Q$ will alter in size and orientation it will remain an e-triangle, thus driving $Q$ onto $S T$ and ensuring that $d(M)=S T$. So $L=S T$ and $M$ is the Fermat/Torricelli/Steiner point of $\triangle W E T$.

Key here is the observation that $\angle E M T$ is $120^{\circ}$. By similar arguments $\angle W M E$ and $\angle W M T$ are also $120^{\circ}$. As "isogonic" is Greek for "equal angles" this is where the name, First Isogonic Center, comes from. But unlike $M, X 13$ will clearly lie outside any triangle containing an obtuse angle in excess of $120^{\circ}$.

What happens to $M$ in such a case? If $\angle W E T>120^{\circ}$ and $P$ lies anywhere in $\triangle W E T$ produce $T E$ to cut one or other of $S Q$ and $Q P$ in $X$. Then $d(P) \geq S X+X E+E T \geq S E+E T=d(E)$ and hence $M$ and $E$ coincide.

Returning to the Old World, how might mathematicians in the British Isles have viewed this confusion over names? It seems likely that the English would have wanted absolutely nothing to do with something that smacked of a French invention. It may be conjectured that the Irish, too, were not terribly interested in $M$ until they came across diagrams of Steiner networks which were full of angles of $120^{\circ}$ and regular hexagons. Although not officially recorded anywhere it was traditional that such diagrams had been realised in Ulster long before the dawn of written history by a chap called MacCool, therefore there seemed a strong case for affording him primacy. It may be further conjectured that the men of Ulster demanded the name MacCool/North whereas the Scots wanted MacCool/East to underline their title to the eastern end of MacCool's creation. So $\mathrm{MacCool} /$ West looks like a committee decision. Maybe the Welsh were a little jealous, claiming that the creation was a surface tension effect of fast-cooling lava and nothing to do with MacCool at all. If so then perhaps it's time for the locals to drop MacCool and simply call $M$ the West Centre?

Whatever the truth of the matter that's the history of Mac$\mathrm{Cool} /$ West and it's now time to establish the isomorphism ... 


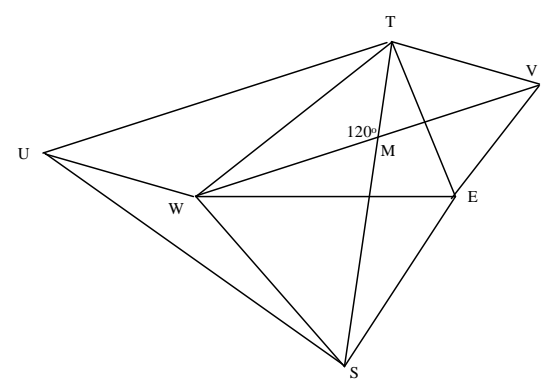

Start again with $\triangle W E T$ (all angles $\leq 120^{\circ}$ ) and construct etriangles $W E S$ and $E T V$ as shown above. Also construct the parallelogram $T U W V$. Note that $S T$ and $V W$ are both of length $L$ and intersect in $M$ at an angle of $120^{\circ}$. Thus $\triangle S T U$ must be an e-triangle of side $L$ because $T U$ and $V W$ have the same length $L$ and $\angle S T U$ being supplementary to $\angle T M W$ is equal to $60^{\circ}$. Since $U W=T V=T E$ and $W S=W E$ the distances from $W$ to the vertices of $\triangle S T U$ are equal to $T E, W E$, and $T W$. As these are precisely the sides of $\triangle W E T$ the connection between Q1 and Q2 is established.

This type of interplay between different areas of mathematics has been used in several joint papers with T.T. West. The initial approach was to treat linear operators as elements of a Banach algebra and derive their best known spectral properties. Then this treatment was reversed to show how all Banach algebras had elements that behaved just like Fredholm and compact operators. In particular the celebrated West decomposition theorem was transplanted to a $B^{*}$ algebra. Next came the application of spectral theory to non-negative matrices with a view to making Perron-Frobenius theory easier to understand. Here there is some evidence that spectral projections have much more to contribute to the study of non-negative matrices than they have done to date.

The last words belong to the Web. There is a third question that has exactly the same answer as Q1 and Q2.

Q3 Find the height $L$ of the largest e-triangle that can be circumscribed about a given triangle with sides $x, y, z$.

Note that $\triangle A B C=\triangle X B C+\triangle X C A+\triangle X A B$ for any point $X$ inside $\triangle A B C$, so the height of any e-triangle equals the sum of the perpendicular distances from any internal point to its three sides. 
Construct the perpendiculars to $W M, E M, T M$ that go through $W, E, T$ respectively. By the isogonality property of $M$ these perpendiculars form an e-triangle that circumscribes $\triangle W E T$ and whose height is $L$. Moreover if $\Delta$ is any other e-triangle that circumscribes $\triangle W E T$ then the perpendicular distances from $M$ to its sides are bounded by $W M, E M, T M$ and so the height of $\Delta$ cannot exceed $L$.

H.W. Kuhn credits Vecten, Professor of Mathématiques Spéciales at the Lycée de Nîmes circa 1800, with being the first to spot this duality. It may indeed be the earliest duality result ever recorded. For related details see "The Ladies Diary or Woman's Almanack" (1755) [p. 47], "Annales de Mathématiques Pures et Appliqués", Vol I (1810-11) [p. 384], and Vol II (1811-12) [p. 91].

Finally in the context of the diagram on the previous page it is interesting to note that if a further e-triangle is constructed on $T W$ then its centroid together with the centroids of $\triangle W E S$ and $\triangle V E T$ forms yet another e-triangle. This is a phenomenon known as Napoleon's Theorem. Apparently Napoleon was a keen amateur mathematician so it is possible that the discovery was truly his, nevertheless he had both Laplace and Lagrange at his beck and call and it would seem likely that one of them may have gifted him this result. All of which begs an intriguing question; where would the world be today if only Napoleon had stuck to his mathematics?

M. R. F. Smyth,

15 Harberton Avenue,

Belfast BT9 6PH

malcolm.smyth@ntlworld.com

Received on 22 January 2006. 